\title{
Avaliação do ciclo de vida no Brasil: uma investigação nas principais bases científicas nacionais
}

\author{
Camila Daniele Willers ${ }^{a *}$, Luciano Brito Rodrigues ${ }^{\mathrm{b}}$, Cristiano Alves da Silva ${ }^{\mathrm{c}}$ \\ a*camiladw@hotmail.com, UESB, Brasil \\ brodrigueslb@gmail.com, UESB, Brasil \\ ccralves@dcdesign.com.br, UFRN, Brasil
}

\begin{abstract}
Resumo
A avaliação do ciclo de vida (ACV) é uma metodologia de avaliação de impacto ambiental de produtos e sistemas de produção considerando todo o ciclo de vida, desde a aquisição de matérias-primas até a disposição final. Este trabalho consistiu na investigação do progresso dos estudos sobre ACV no Brasil, por meio de uma pesquisa bibliográfica em eventos e periódicos oficiais ou reconhecidos pela Associação Brasileira de Engenharia de Produção e na base de dados SciELO Brasil. Foram identificados 80 artigos, a maioria de instituições das regiões Sul e Sudeste. A Universidade de São Paulo (USP) e Universidade Federal de Santa Catarina (UFSC) apresentaram o maior número de publicações dentre as 50 instituições identificadas. Verificou-se que 17 artigos aplicaram efetivamente a metodologia ACV em um estudo de caso, sendo que 11 utilizaram a metodologia para avaliar processo produtivo e 6 para comparar materiais ou processos.
\end{abstract}

Palavras-chave

Avaliação do ciclo de vida. Avaliação de impacto. ISO 14040. Pesquisa bibliográfica.

\section{Introdução}

0 modo como a questão ambiental está em evidência tem fortalecido seus valores e imposto às indústrias novos desafios para atender a demanda de um mercado consumidor com crescente interesse na forma como os produtos e serviços são produzidos, utilizados e descartados e em como esses afetam o meio ambiente. As indústrias também tem se interessado igualmente pela cobrança de práticas de produção mais limpas das grandes organizações-parceiras, pelas certificações com reconhecimento internacional e pelo escasseamento dos recursos naturais etc. (OLIVEIRA; SERRA, 2010).

Esse novo comportamento demandou o desenvolvimento de abordagens e ferramentas de gestão que possibilitassem às empresas avaliar as consequências ambientais das decisões que tomavam em relação aos seus processos ou produtos. Esta tarefa mostrou-se extremamente complexa em função da necessidade de estabelecimento de critérios comuns de comparação e ainda da criação de uma abordagem completa do que se passou a chamar o ciclo de vida do produto. Assim, desenvolveu-se a ferramenta de avaliação do ciclo de vida.

A avaliação do ciclo de vida (ACV) é uma abordagem do "berço ao túmulo" para a avaliação de sistemas de produção (EPA e SETAC, 2006). Nessa óptica, a avaliação começa com a aquisição de matérias-primas e termina no momento em que todos os materiais sofrem disposição final. A ACV permite a estimativa dos impactos ambientais cumulativos resultantes de todas as fases do ciclo de vida do produto, muitas vezes incluindo impactos não considerados em análises mais tradicionais (por exemplo, a extração de matérias-primas, material de transporte, disposição final do produto etc.) (GIANNETTl et al., 2008). Ao incluir os impactos ao longo do ciclo de vida do produto, a ACV oferece uma visão abrangente dos aspectos ambientais do produto ou processo e uma imagem mais precisa do verdadeiro trade-off (balanço entre prós e contras) do produto e da seleção de 
processos. A Figura 1 ilustra as possíveis etapas do ciclo de vida que podem ser consideradas em uma ACV e as entradas e saídas típicas.

A ACV é padronizada pela International Organization for Standardization (ISO), que elaborou a ISO 14040 sobre a Avaliação do Ciclo de Vida, publicada no Brasil pela Associação Brasileira de Normas Técnicas, ABNT. De acordo com a ISO 14040 (2006), a ACV é a compilação e avaliação das entradas, das saídas e dos impactos ambientais potenciais de um sistema de produto ao longo do seu ciclo de vida. A metodologia de ACV é divida em fases: definição de objetivo e escopo, análise de inventário, avaliação de impactos e interpretação de resultados.

Objetivo: Deve declarar inequivocamente a aplicação pretendida, as razões para conduzir o estudo e o público-alvo;

Escopo: Devem ser considerados e claramente descritos os seguintes itens: a) função e unidade funcional; b) fronteiras do sistema; c) requisitos da qualidade dos dados; d) comparações entre sistemas; e) considerações sobre análise crítica;

Análise de inventário: Envolve a coleta de dados e procedimentos de cálculo para quantificar as entradas e saídas pertinentes de um sistema de produto. Este consiste em um balanço de energia e massa que configura o inventário de ciclo de vida (ICV), cuja análise avalia os efeitos ambientais do sistema;

Avaliação de impactos: É dirigida à avaliação da significância dos impactos ambientais potenciais, usando os resultados da análise de inventário do ciclo de vida. Em geral, esse processo envolve a associação de dados do inventário com impactos ambientais específicos e a tentativa de compreender esses impactos;

Interpretação de resultados: Fase onde as constatações da análise do inventário e da avaliação de impacto - ou, no caso de estudos de inventário do ciclo de vida (ICV), somente os resultados da análise de inventário - são combinadas, de forma consistente, com o objetivo e o escopo definidos, visando alcançar conclusões e recomendações.

As áreas de atuação para a ACV são inúmeras. Segundo a ISO 14040 (2006), algumas são: avaliação de risco, avaliação de desempenho ambiental, auditoria ambiental e avaliação de impacto ambiental.

A ACV, cujas origens remontam à década de 1960, encontra-se difundida em muitos países, com estudos diversos, os quais têm contribuído para a consolidação da metodologia em todo o mundo. As principais referências na área, devido ao pioneirismo e alto nível dos estudos desenvolvidos, são norte americanas e europeias. No Brasil, pode-se considerar como início da história da ACV a década de 1990,

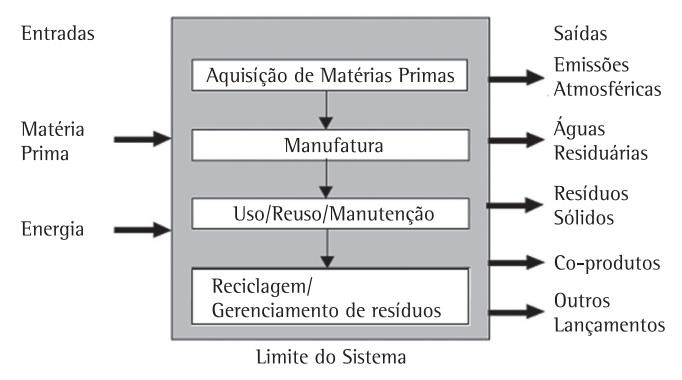

Figura 1. Estágios do ciclo de vida (EPA e SETAC, 2006).

com a criação do subcomitê da ABNT, o qual passou a integrar o Comitê Técnico TC 207 da 1SO, que trabalhou na elaboração das normas da família $1 S 0$ 14000 (CHEHEBE, 1997; SANTOS, 2006).

Em 2002 foi fundada a Associação Brasileira de Ciclo de Vida, ABCV, a qual vem se dedicando à disseminação e consolidação da ACV no Brasil, principalmente pela promoção de conferências, destacando-se três delas. A primeira ocorreu em 2007, em São Paulo: a segunda edição da Conferência Internacional sobre Avaliação do Ciclo de Vida, CILCA, agora denominada Conferência Internacional sobre Análise do Ciclo de Vida na América Latina. A ABCV também vem promovendo o Congresso Brasileiro sobre Gestão do Ciclo de Vida de Produtos e Serviços, CBGCV, com duas edições, em 2008 e 2010. Evento bianual, apesar de recente, ele já pode ser considerado o principal evento nacional dedicado ao tema ACV. Entretanto, ainda encontra-se em fase de consolidação, o que pode ser verificado pelo número de trabalhos apresentados nas duas primeiras edições (44 e 55, respectivamente), os quais não são, necessariamente, relacionados a ACV qualitativas. A terceira edição do Congresso ocorre em setembro de 2012.

Já é possivel perceber a estruturação e organização envolvendo o tema ACV o Brasil. 0 país oferece um vasto campo de oportunidades para aplicação da metodologia, seja em setores da indústria e agroindústria, seja na área acadêmica, cujos objetivos estão voltados para o desenvolvimento de estudos e pesquisas visando a solução de problemas na área ambiental, com possibilidade de aplicação dos resultados nos setores produtivos citados.

Considerando que os resultados produzidos pela área acadêmica são divulgados preferencialmente na forma de artigos científicos, em conferências e periódicos, este trabalho teve como objetivo investigar o progresso dos estudos sobre avaliação do ciclo de vida desenvolvidos no Brasil a partir de uma pesquisa nas principais bases de dados nacionais de acesso livre onde o tema ACV pudesse ser divulgado.

Lima (2007), em seu trabalho de mestrado, fez uma pesquisa bibliográfica sobre ACV considerando como sua principal base de dados os periódicos internacionais 
da base Science Direct. Para os trabalhos nacionais, a autora utilizou como base de dados o banco de teses e dissertações da Coordenação de Aperfeiçoamento de Pessoal de Nível Superior (Capes) e o banco de dados do Instituto Brasileiro de Informação em Ciência e Tecnologia (IBICT), nos quais se encontram as teses e dissertações defendidas junto aos programas de pós-graduação do país. Face ao excelente trabalho da autora, percebe-se que não foi realizada uma busca mais específica nas bases nacionais contemplando os artigos científicos publicados periódicos e congressos brasileiros.

\section{Metodologia}

Uma vez que o Brasil ainda não possui um periódico específico sobre o tema, nem mesmo uma conferência historicamente consolidada (mesmo com o recente (BGCV), este trabalho primeiramente considerou como base de dados para pesquisa as principais conferências e periódicos oficiais da Associação Brasileira de Engenharia de Produção, ABEPRO, instituição que há mais de 20 anos congrega docentes, discentes, pesquisadores e profissionais da área de Engenharia de Produção. A ABEPRO estabelece, dentre as áreas de atuação da Engenharia de Produção, a Engenharia da Sustentabilidade, na qual trabalhos sobre ACV podem ser considerados, tendo em vista as subáreas relacionadas.

A pesquisa, portanto, teve como fontes:

- Os anais dos Encontros Nacionais de Engenharia de Produção de 1996 a 2010, disponíveis na página do evento no endereço http://publicacoes.abepro. org.br/;

- A Revista Produção Online, ISSN 1676-1901, de 2008 a julho de 2011, disponível no endereço http:// producaoonline.org.br/index.php/rpo/search;

- 0 Brazilian Journal of Operations \& Production Management (BJO\&PM), ISSN 1679-8171, de 2004 a julho de 2011, disponível no endereço http://www. abepro.org.br/bjopm/index.php/bjopm/index; e

•A Revista Produção, ISSN 0103-6513, de 1991 a 2011, disponível no endereço http://www.scielo. $\mathrm{br} / \mathrm{sciel}$.php?script=sci_issues\&pid=0103-6513\& lng $=$ pt\&nrm=iso.

Uma vez que a Revista Produção pertence à base de dados SciELO Brasil, a qual contém outros periódicos nacionais de áreas diversas, sendo alguns afins da engenharia de produção, e que a ferramenta desta base permite uma pesquisa ampliada em todos os periódicos nela contidos, a pesquisa nesse caso foi expandida para toda a base de dados. A pesquisa considerou os artigos publicados até julho de 2011, disponíveis no endereço http://www.scielo.br/?lng=pt.
Considerando a recente iniciativa da ABEPRO, com a criação do Selo ABEPRO de reconhecimento de periódicos científicos institucionais, a pesquisa também incluiu a Revista Gestão Industrial, ISSN 1808-0448, de 2005 a 2011, contemplada em 2010 com o referido selo, disponível em http://www.pg.utfpr. edu.br/depog/periodicos/index.php/revistagi/search.

Uma vez definidas as bases para consulta, procedeu-se à pesquisa, que foi realizada em forma de palavras-chave, fundamentando a escolha dessas em assuntos que poderiam estar relacionados direta ou indiretamente com a ACV, e, nas questões semânticas de idiomas estrangeiros, visto que as fontes consultadas possuem trabalhos em português, inglês e espanhol. As palavras selecionadas foram: Life cycle, ACV, LCA, ciclo de vida, $1 \mathrm{SO}$ 14040, Ecodesign, $\mathrm{P}+\mathrm{L}$, produção mais limpa e produção limpa. Nos primeiros resultados obtidos por meio desta busca foi realizado um processo de seleção para identificar somente os trabalhos que citavam a ACV em seu contexto. Em seguida a pesquisa selecionou os trabalhos que aplicaram a ACV em estudo de caso.

\section{Resultados e discussão}

A pesquisa identificou 80 artigos que citavam ACV, sendo 63 do ENEGEP, 12 da SciELO Brasil, 5 da Revista Produção Online e nenhum nos periódicos BJO\&PM e Gestão Industrial. A leitura permitiu estruturar os resultados deste trabalho em três itens: instituições de pesquisa e base de dados; áreas de atuação; metodologia empregada na aplicação da ACV, abordados com base em dados empíricos claros e inequívocos, discutidos individualmente a seguir.

Os resultados para os dois primeiros itens serão apresentados e discutidos para todos os trabalhos encontrados, enquanto que o último será discutido somente com as publicações que aplicaram a metodologia da ACV em estudo de caso.

\subsection{Instituições de pesquisa e base de dados}

Foram identificadas as instituições que realizam investigações na área da ACV, ou mesmo que abordaram o assunto indiretamente em suas pesquisas. Sendo assim, a pesquisa contabilizou 41 universidades ou instituições de pesquisa e 9 empresas, consultorias ou instituições estrangeiras. Em relação à frequência com que as instituições constavam em cada artigo, foram identificadas 107 participações de instituições em um total de 80 artigos selecionados, sendo 96 delas de universidades, e 11 participações de autores vinculados a empresas, ou instituições estrangeiras. A Figura 2 apresenta as universidades que foram identificadas com uma frequência mínima de três participações em diferentes trabalhos publicados. 
As universidades que possuíam maior número de publicações foram a USP (Universidade de São Paulo) e a UFSC (Universidade Federal de Santa Catarina), com 11 e 7 artigos, respectivamente, seguidas pela Unisinos (Universidade do Vale do Rio dos Sinos), com

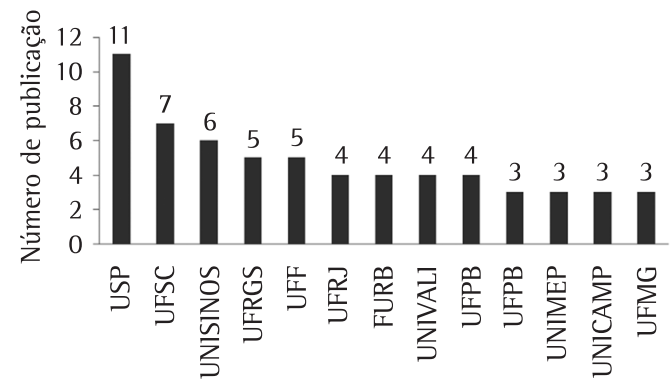

Universidades

Figura 2. Número de publicações sobre ACV produzidas por universidades brasileiras nas bases de dados consultadas.

Participação das Universidades

(a) no ENEGEP, no Scielo Brasil e na Revista de Produção Online

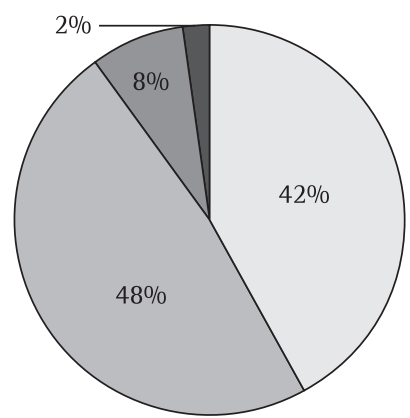

$\square$ Sul $\square$ Sudeste $\square$ Nordeste $\square$ Norte

Participação das Universidades na Revista de Produção Online

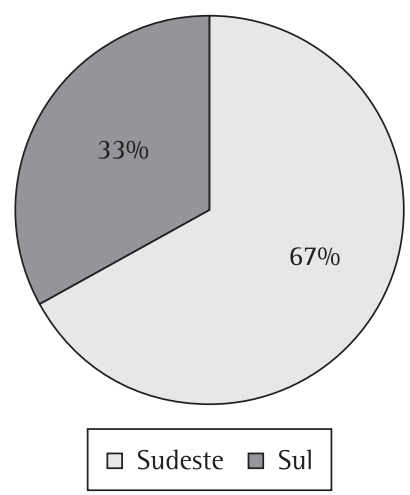

6 artigos, UFRGS (Universidade Federal do Rio Grande do Sul) e UFF (Universidade Federal Fluminense), com 5 publicações, pela UFRJ (Universidade Federal do Rio de Janeiro), FURB (Universidade Regional de Blumenau), Univali (Universidade do Vale do Itajaí) e UFPB (Universidade Federal da Paraíba), com 4 artigos cada uma, e a UFSCar (Universidade Federal de São Carlos), a Unimep (Universidade Metodista de Piracicaba), a Unicamp (Universidade Estadual de Campinas) e a UFMG (Universidade Federal de Minas Gerais), com 3 artigos cada.

Evidencia-se maior concentração das publicações nas regiões Sudeste e Sul, o que foi verificado a partir do número total das participações das universidades, com o qual se obteve a distribuição geográfica dessas no Brasil (Figura 3a). Para elaboração desse gráfico foram excluídos os 11 trabalhos vinculados às empresas e consultorias, visto que estas podem estar presentes

(b)

Participações das Universidades no Scielo Brasil

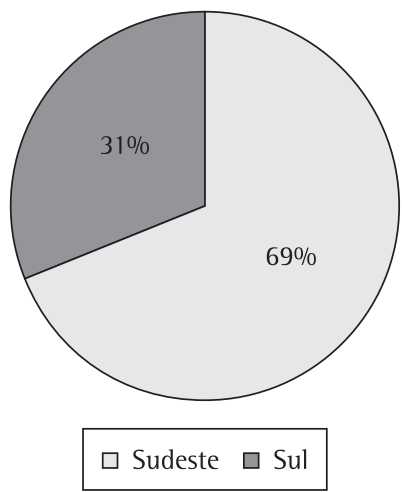

Participação das Universidades no ENEGEP

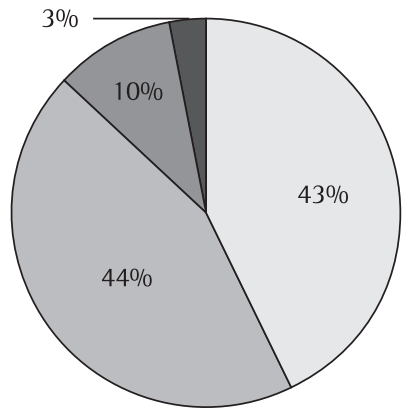

$\square$ Sul $\square$ Sudeste $\square$ Nordeste $\square$ Norte

Figura 3. Distribuição por região do país das participações das universidades em trabalhos selecionados na pesquisa bibliográfica sobre a avaliação do ciclo de vida (a) participação total das universidades nos trabalhos das três bases de dados, (b) participação das universidades nos trabalhos na base de dados da SciELO Brasil, (c) participação das universidades nos trabalhos da Revista Produção Online, (d) participação das universidades nos trabalhos dos anais do ENEGEP. 
em mais de uma região do território nacional ou até mesmo em outros países.

De acordo com a Figura 3a, a região Sudeste é a que apresenta maior produção científica na área, com a frequência de 46 participações de suas universidades no total de 96 , seguida pela região Sul, com 40. A maior parte dessas publicações da região Sudeste está relacionada a programas de pós-graduação da USP, UFRJ, UFF e Unimep. Destacam-se na região Sul os trabalhos da UFSC, representada pelo Grupo de Pesquisas em Avaliação do Ciclo de Vida da UFSC (2010), criado em 2006.

Dentre as universidades com maior frequência de participação na publicação de artigos, tem-se na região Norte, que responde por $2 \%$ das publicações encontradas, a Universidade Federal do Pará (UFPA), com 100\% das participações de sua região, equivalente à participação em dois trabalhos diferentes. No Nordeste, que responde por $8 \%$ das publicações, destaca-se a UFPB com 50\%, equivalente a 4 participações em trabalhos diferentes. Na região Sudeste, que responde por $48 \%$ das publicações, destaca-se a USP com 24\%, equivalente a 10 participações em trabalhos. E, na região Sul, que responde por $42 \%$ das publicações encontradas, destaca-se a UFSC, com 18\%, equivalente a 7 participações em trabalhos.

Constata-se que, em relação à divulgação de trabalhos, o ENEGEP é de fato o principal evento de Engenharia de Produção do Brasil devendo, portanto, intensificar a divulgação dos trabalhos sobre ACV. Esse evento foi o meio que mais divulgou trabalhos sobre ACV dentre as bases consultadas, concentrando 63 dos 80 artigos selecionados pela pesquisa, seguido pela SciElO Brasil, com 12 artigos e, por último, a Revista Produção Online, com 5 artigos (Figura 4). Contudo, ao analisar a participação das regiões em cada periódico e evento, pelas Figuras 3b, c, d, constata-se tendência das regiões Sudeste e Sul a ter maior predominância na participação, enquanto as demais - Centro-Oeste, Norte e Nordeste - a deter menor participação no ENEGEP e nem constar na Revista Produção Online e na base de dados SciElO Brasil. De modo geral, pode-se afirmar que as regiões Centro-Oeste, Norte e Nordeste se caracterizam pela baixa procura por esses meios científicos para a divulgação de trabalhos.

Em relação à base de dados SciELO Brasil, foram identificados 12 artigos de 10 periódicos diferentes, sendo seis da área de Engenharia, e os demais de áreas das Ciências Exatas e da Terra, das Ciências Agrárias e das Ciências Humanas. Os periódicos Produção (ISSN 0104-530X) e Gestão \& Produção (ISSN 1980-5411 - versão on-line) são os que possuem maior número de artigos sobre ACV - dois cada. Ressalta-se que estes são os dois principais periódicos
Artigos selecionados

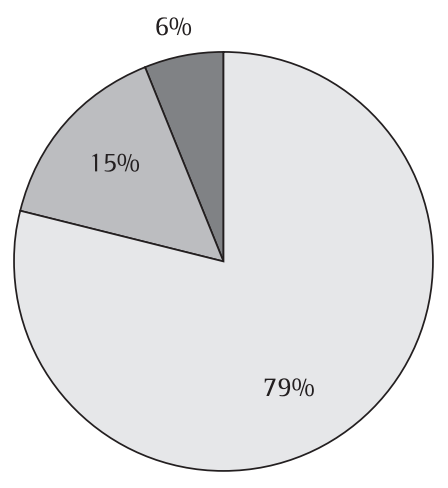

$\square$ ENEGEP $\square$ Scielo $\square$ Revista Produção Online

Figura 4. Total de artigos selecionados pela pesquisa bibliográfica sobre avaliação do ciclo de vida nos anais do ENEGEP de 1996 a 2010, na base de dados da Revista Produção Online de 2008 a julho de 2011 e na base de dados SciElO Brasil até julho de 2011.

nacionais da área de Engenharia de Produção, segundo a classificação da Coordenação de Aperfeiçoamento de Pessoal de Nível Superior (Capes), o Qualis, em que os mesmos estão classificados com estrato B2 para a área de Engenharias 111, onde a Engenharia de Produção está incluída.

Dos 12 periódicos, apenas dois não possuem no Qualis estrato para a área de Engenharias 111, estando os demais classificados como B1 ou B2, mostrando a qualidade dos mesmos para a área (Tabela 1).

Dessa maneira foi evidenciada a presença da temática da gestão ambiental, principalmente nas Engenharias, e igualmente a abordagem da metodologia de ACV nesta área, confirmando a escolha feita neste artigo, pelos eventos e periódicos da área de Engenharia de Produção.

\section{2. Áreas de atuação}

Neste tópico foram identificadas as principais áreas em que a ACV é aplicada. Desse modo, das 80 publicações encontradas, excluindo-se as 17 que aplicaram a metodologia de ACV a um estudo de caso, as demais concentram seus temas na utilização da ACV como ferramenta de gestão ambiental. Dentro deste tema existiram alguns objetivos específicos relacionados ao conceito de emissão zero (ROQUE; MOURA JÚNIOR, 2007), produção mais limpa (SILVA; SILVA, 1998), logística reversa (CARDOSO et al., 2007; GONÇALVES; MARINS, 2006; AZEVEDO; CARMO; FERREIRA, 2010), sustentabilidade ambiental das atividades (LIBRELOTTO; FERROLI; RADOS, 2003; FARIAS FILHO et al., 2005; PATZLAFF; KERN; GONZÁLEZ, 2010) e sistemas de informação (ÑAURI et al., 1997). 
Tabela 1. Periódicos selecionados pela pesquisa bibliográfica sobre avaliação do ciclo de vida na base de dados SciELO Brasil, abrangendo os artigos publicados até julho de 2011.

\begin{tabular}{|c|c|c|c|}
\hline Nome do periódico & $\begin{array}{l}\mathrm{N}^{0} \text { de artigos } \\
\text { encontrados }\end{array}$ & Assunto & $\begin{array}{c}\text { Qualis para } \\
\text { Engenharias } 111^{*}\end{array}$ \\
\hline Produção & 2 & Engenharias & B2 \\
\hline Gestão \& Produção & 2 & Engenharias & B2 \\
\hline Rem: Revista Escola de Minas & 1 & Engenharias e Ciências Exatas e da Terra & B2 \\
\hline Brazilian Journal of Chemical Engineering & 1 & Engenharias e Ciências Exatas e da Terra & B1 \\
\hline Química Nova & 1 & Ciências Exatas e da Terra & $\mathrm{B} 1$ \\
\hline Revista Brasileira de Zootecnia & 1 & Ciências Agrárias & - \\
\hline Ciência e Rural & 1 & Ciências Agrárias & B2 \\
\hline Ambiente \& Sociedade & 1 & Ciências Humanas & - \\
\hline Revista Polímeros & 1 & Engenharias e Ciências Exatas e da Terra & B2 \\
\hline Revista Engenharia Sanitária e Ambiental & 1 & Engenharias & B2 \\
\hline Total & 12 & & \\
\hline
\end{tabular}

*Classificação realizada pela Capes em fevereiro de 2010.

Alguns trabalhos abordaram a ACV para concepção de produtos no conceito de ecodesign (MAKIYA, 2007; BORCHARDT et al., 2007, 2008, 2010a, b; GUELERE FILHO et al., 2008) ou na concepção de projetos envolvendo a variável ambiental (FERROLl et al., 1997; SANTOS, 1998a; POZZOBON; RUPPENTHAL, 1999). Encontra-se a citação de ACV em artigos sobre o processo de rotulagem ambiental (SANTOS, 1998b; ALMEIDA; BRUNSTEIN, 1998), avaliação de impactos ambientais (MACHADO; HOFFMANN, 2003; SELLITTO; BORCHARDT; PEREIRA, 2010) e balanço ambiental (MONDARDO FILHO; FRANK, 2000).

Alguns textos sobre educação ambiental sugeriram a introdução da ACV nas ementas dos cursos de engenharia ou disciplinas com temática ambiental para auxiliar o profissional nos processos de tomada de decisão (JABBOUR, 2006; ULIANA, 2008). Outros autores discutiram a metodologia ACV, seu contexto histórico e conceitos, ou ainda realizaram uma revisão bibliográfica e análise crítica da mesma (ABARCA et al., 1997; LOBO; LIMA, 1997; MAGALHÃES, 1998; BARBOSA JÚNIOR et al., 2007; RUY; ALLIPRANDINI, 2010; CULTRI; SAAVEDRA; OMETTO, 2010; WILLERS et al., 2010). A questão dos softwares disponíveis para a ACV foi, também, abordada, e comparações entre eles foram realizadas, ressaltando os recursos que cada um oferece (RODRIGUES et al., 2008).

Temas como a relação entre o marketing ambiental e a ACV também foram discutidos, também como a ACV pode fornecer dados, desde a concepção do produto até o descarte, indicando oportunidades de melhorias contínuas no processo ou no produto, atendendo, assim, preceitos de responsabilidade socioambiental do mercado (BARRETO et al., 2007). Outros se detiveram apenas na citação do ACV, não esclarecendo o conceito, suas propriedades ou outras características da metodologia (GIESTA; SILVA, 2006; PASQUAL; COSTA; FERNANDES, 2006; CORAL; STROBEL; SELIG, 2004; TEIXEIRA; TEIXEIRA,
1997; TOLEDO; TURRIONI; BALESTRASSI, 2003; SILVA FILHO; SICSÚ, 2003; KRAEMER, 2004; DETONI; PEREIRA, 2005; PINTO et al., 2007; MADRUGA; NASCIMENTO; ZAWISLAK, 1999; BIAZIN; GODOY, 2000; PASCHOALINO; MARCONE; JARDIM, 2010; AZEVEDO; NOLASCO, 2009; TÁVORA; QUELHAS, 2003).

Na Figura 5 pode-se verificar a frequência com que os temas citados anteriormente foram abordados.

\subsection{Metodologia empregada na aplicação da $A C V$}

Esta parte do estudo tratou de analisar os artigos que aplicaram a metodologia de ACV a um estudo de caso. Visto que a maioria das metodologias empregadas nas publicações é baseada na norma ISO 14040 (2006), a pesquisa utilizou-a como referência para análise dos trabalhos, os quais são apresentados no Quadro 1.

Pode-se observar nesses artigos o tipo de ACV aplicada, sendo que 11 utilizaram a metodologia para avaliar o processo produtivo e 6 para comparar materiais ou processos. Constou-se, ainda, que 10 trabalhos não utilizaram nenhum software para aplicar a metodologia, demonstrando que não há uma relação de limitação ou dependência que exija a aquisição das licenças para aplicar a ACV.

Quanto à metodologia aplicada, foram identificadas três publicações que citaram metodologias distintas (artigos números 1, 4 e 16, Quadro 1). No entanto, essas metodologias se baseiam indiretamente na ISO 14040 (2006) e se tornaram documentos guias determinando os princípios e práticas da ACV.

Aprofundando a análise, 11 artigos não aplicaram todas as fases da metodologia, como no caso do refino do petróleo, que utilizou os princípios da ACV para desenvolver um software específico para refinarias (artigo número 5, Quadro 1); o estudo sobre sacos plásticos apenas modela o sistema para aplicação 


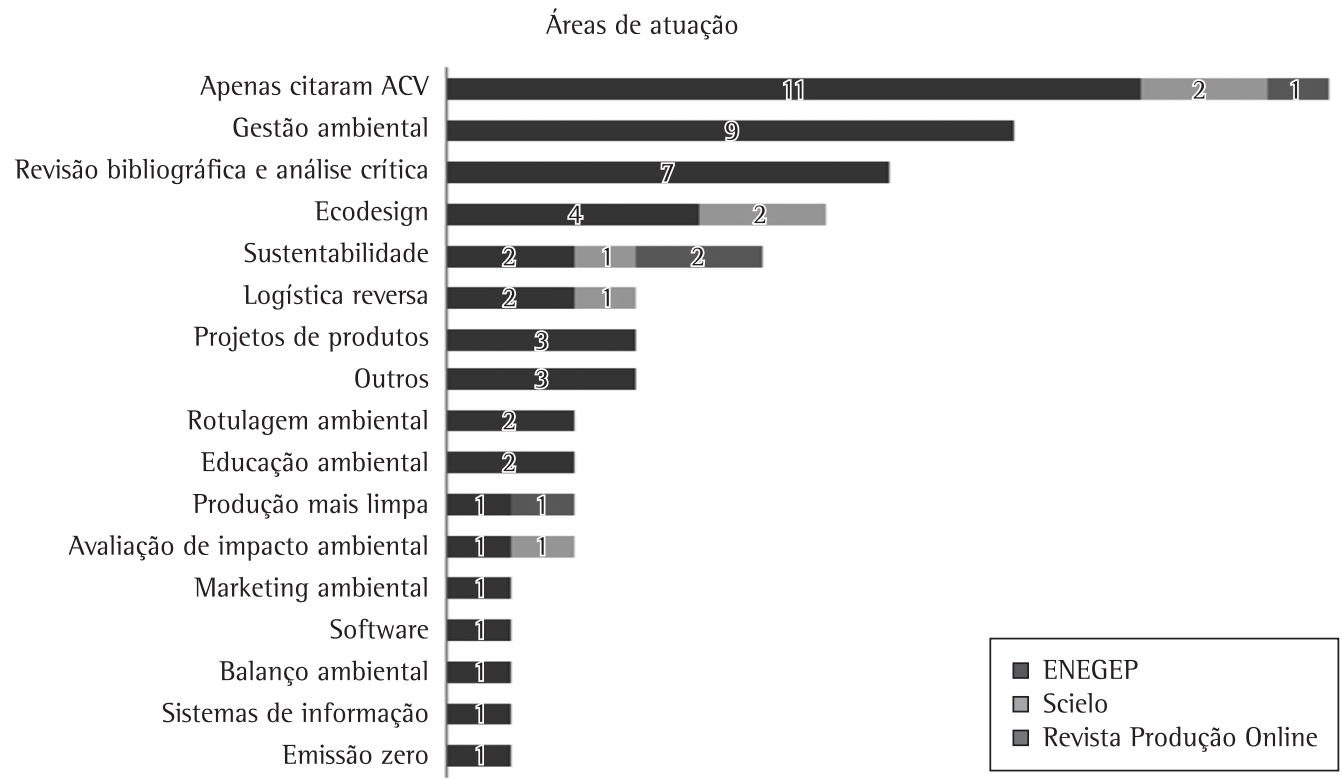

Figura 5. Número de artigos selecionados em cada base de dados, relacionando as áreas em que a avaliação do ciclo de vida foi citada, fundamentados na pesquisa bibliográfica realizada nos anais do ENEGEP de 1996 a 2010, na base de dados SciEL0 Brasil, abrangendo os artigos publicados até julho de 2011, e na Revista Produção Online, de 2008 a julho de 2011.

da ACV (artigo número 6, Quadro 1). A pesquisa identificou ainda que seis artigos que realizaram a ACV até as etapas de análise de inventário de ciclo de vida e interpretação dos resultados, e, mesmo assim, obtiveram resultados significantes (artigos números 2, 3, 12, 13, 15 e 16, Quadro 1).

\section{Considerações finais}

A pesquisa identificou um total de 80 artigos, sendo que 63 se restringiram a uma abordagem conceitual da ACV, sem uma aplicação efetiva da metodologia. Tomando-se por base os anais do ENEGEP, a base de dados SciELO Brasil e a Revista Produção Online, foi possível conhecer as instituições que têm apresentado maior participação na produção científica em relação ao tema, bem como a região de origem no país. Destacam-se a USP e a UFSC com o maior número de publicações dentre as instituições. As regiões Sul e Sudeste concentram a maior produção de trabalhos no país, não apenas pelas instituições já citadas, mas também devido aos trabalhos da Unisinos, UFRGS, UFF, UFRJ, FURB e Univali, mostrando assim que os estudos sobre ACV encontram-se distribuídos entre várias instituições em ambas as regiões.

Os trabalhos das regiões Norte e Nordeste são predominantemente da UFPA e da UFPB que, percentualmente, são as instituições que possuem maior número de participação nas publicações para a região, com 100\% e 50\% dos trabalhos produzidos, respectivamente. Em seguida tem-se a USP, com 24\% dos trabalhos da região Sudeste, e a UFSC, com 18\% dos trabalhos da região Sul. Ressalta-se, ainda, a ausência de participação de instituições da região Centro-Oeste bem como o baixo número de participação de instituições das regiões Norte e Nordeste. lsso pode ser atribuído a baixa procura pelos periódicos e evento estudados na presente pesquisa ou a baixa disseminação da metodologia de ACV nessas regiões.

Entre as bases de dados pesquisadas, evidenciou-se a preferência dos autores pela publicação de trabalhos sobre ACV no ENEGEP, com 79\% dos artigos, seguido pela SciElO Brasil, com 15\%, a Revista Produção Online, com 6\%, enquanto que não foram encontrados trabalhos relacionados à ACV no BJO\&PM e na Revista Gestão Industrial. Em relação à base de dados SciELO Brasil foi verificado que a maior parte dos artigos constava em periódicos da área de Engenharia, dentre eles, os dois principais periódicos da área de Engenharia de Produção, confirmando a escolha feita neste artigo, relacionada à opção preferencial por eventos e periódicos desta área. Entretanto, há a ausência de um periódico nacional específico que aborde o assunto de ACV, a exemplo do The International Journal of Life Cycle Assessment, de modo a incentivar e formar referenciais científicos para os estudos e a sua divulgação no país. 
Quadro 1. Classificação dos artigos selecionados na pesquisa bibliográfica realizada nos anais do ENEGEP de 1996 a 2010 , na SciELO Brasil até julho de 2011, e na Revista Produção Online de 2008 a julho de 2011 conforme ISO 14040 (2006).

\begin{tabular}{|c|c|c|c|c|c|}
\hline & Autor & Estudo & Metodologia & Fase aplicada & Software \\
\hline 1 & $\begin{array}{l}\text { Graf e Figueiredo } \\
\text { (1999) }\end{array}$ & $\begin{array}{c}\text { ACV comparativa da produção } \\
\text { de alface com as técnicas } \\
\text { intensiva, hidropônica e } \\
\text { orgânica }\end{array}$ & Curran (1996) & $\begin{array}{l}\text { Objetivo e escopo, análise de } \\
\text { inventário, avaliação de impactos e } \\
\text { interpretação de resultados }\end{array}$ & ---- \\
\hline 2 & $\begin{array}{l}\text { Ferreira e Frank } \\
\qquad(2000)\end{array}$ & $\begin{array}{l}\text { ACV comparativa entre a } \\
\text { chapa de aço inoxidável e a } \\
\text { chapa de aço carbono com } \\
\text { aplicação do } \\
\text { autolimpante }\end{array}$ & $\begin{array}{l}\text { 1SO 14040, através de } \\
\text { Check list }\end{array}$ & $\begin{array}{c}\text { Análise de inventário e interpretação } \\
\text { de resultados }\end{array}$ & --- \\
\hline 3 & $\begin{array}{l}\text { Bastos e Possamai } \\
\text { (2002) }\end{array}$ & $\begin{array}{l}\text { ACV comparativo no processo } \\
\text { produtivo de tecidos de malha }\end{array}$ & $\begin{array}{l}\text { ISO } 14040 \text { e Chehebe } \\
\text { (1997) }\end{array}$ & $\begin{array}{l}\text { Objetivo e escopo, análise de } \\
\text { inventário e interpretação de } \\
\text { resultados }\end{array}$ & ---- \\
\hline 4 & $\begin{array}{c}\text { Jacovelli e } \\
\text { Figueiredo (2003) }\end{array}$ & $\begin{array}{l}\text { ACV simplificada aplicada à } \\
\text { evolução de tornos }\end{array}$ & $\begin{array}{l}\text { Graedel, Allenby and } \\
\text { Comrie (1995) }\end{array}$ & $\begin{array}{l}\text { Análise de inventário, avaliação } \\
\text { de impactos e interpretação de } \\
\text { resultados }\end{array}$ & ---- \\
\hline 5 & $\begin{array}{l}\text { Ugaya e Henschel } \\
\qquad(2004)\end{array}$ & $\begin{array}{l}\text { Desenvolvimento de software } \\
\text { de ACV para o processo de } \\
\text { refino de petróleo }\end{array}$ & ISO 14040 & $\begin{array}{c}\text { Análise de inventário e avaliação de } \\
\text { impactos }\end{array}$ & RefACV \\
\hline 6 & $\begin{array}{l}\text { lbrahim et al. } \\
\qquad(2007)\end{array}$ & $\begin{array}{l}\text { Modelo de ACV de sacos } \\
\text { plásticos de polietileno de } \\
\text { baixa densidade }\end{array}$ & 1S0 14040 & Objetivo e escopo & Umberto \\
\hline 7 & $\begin{array}{l}\text { Almeida et al. } \\
\text { (2008) }\end{array}$ & $\begin{array}{c}\text { ACV da indústria processadora } \\
\text { de aves }\end{array}$ & ISO 14040 & $\begin{array}{l}\text { Objetivo e escopo, análise de } \\
\text { inventário, avaliação de impactos }\end{array}$ & SimaPro \\
\hline 8 & Silva (2009) & $\begin{array}{l}\text { ACV do transporte de tampas } \\
\text { de plástico usadas em } \\
\text { dispositivos eletrônicos }\end{array}$ & ISO 14040 & $\begin{array}{l}\text { Objetivo e escopo, análise de } \\
\text { inventário, avaliação de impactos e } \\
\text { interpretação de resultados }\end{array}$ & ---- \\
\hline 9 & $\begin{array}{l}\text { Nigri, } \\
\text { Romeiro Filho e } \\
\text { Rocha (2009) }\end{array}$ & ACV do cimento tipo portland & 1S0 14040 & $\begin{array}{c}\text { Objetivo e escopo, análise de } \\
\text { inventário, avaliação de impactos e } \\
\text { interpretação de resultados }\end{array}$ & ---- \\
\hline 10 & $\begin{array}{l}\text { Santos e Tenório } \\
\text { ( 2010) }\end{array}$ & $\begin{array}{c}\text { ACV de evaporadores para } \\
\text { usinas de } \\
\text { cana-de-açúcar }\end{array}$ & 1S0 14040 & $\begin{array}{l}\text { Objetivo e escopo, análise de } \\
\text { inventário, avaliação de impactos e } \\
\text { interpretação de resultados }\end{array}$ & ---- \\
\hline 11 & $\begin{array}{l}\text { Bauer e } \\
\text { Maciel Filho (2004) }\end{array}$ & $\begin{array}{l}\text { ACV da produção de } \\
\text { propileno glicol }\end{array}$ & ISO 14040 & $\begin{array}{l}\text { Escopo, análise de inventário, } \\
\text { avaliação de impactos }\end{array}$ & $\begin{array}{l}\text { HYSYS.Plant } \\
\text { v. } 2.4\end{array}$ \\
\hline 12 & $\begin{array}{l}\text { Giannetti et al. } \\
\text { (2008) }\end{array}$ & $\begin{array}{c}\text { Inventário de ciclo de vida } \\
\text { da manufatura de seringas } \\
\text { odontológicas }\end{array}$ & ISO 14040 & $\begin{array}{l}\text { Objetivo e escopo, análise de } \\
\text { inventário e interpretação de } \\
\text { resultados }\end{array}$ & --- \\
\hline 13 & Nunes et al. (2010) & $\begin{array}{l}\text { Inventário de ciclo de vida } \\
\text { e sua análise na produção } \\
\text { farmacêutica }\end{array}$ & 1S0 14040 & $\begin{array}{l}\text { Objetivo e escopo, análise de } \\
\text { inventário e interpretação de } \\
\text { resultados }\end{array}$ & Umberto \\
\hline 14 & Nigri et al., 2010 & $\begin{array}{c}\text { ACV simplificada e } \\
\text { comparativa da produção de } \\
\text { cachaça artesanal e industrial }\end{array}$ & ISO 14040 & $\begin{array}{l}\text { Objetivo e escopo, análise de } \\
\text { inventário, avaliação de impactos e } \\
\text { interpretação de resultados }\end{array}$ & ---- \\
\hline 15 & $\begin{array}{l}\text { Queiroz e Garcia } \\
\qquad(2011)\end{array}$ & $\begin{array}{l}\text { Inventário de ciclo de vida de } \\
\text { sacolas plásticas de polietileno }\end{array}$ & ISO 14040 & $\begin{array}{l}\text { Objetivo e escopo, análise de } \\
\text { inventário e interpretação de } \\
\text { resultados }\end{array}$ & PEMS \\
\hline 16 & $\begin{array}{l}\text { Garcia e Von } \\
\text { Sperling (2010) }\end{array}$ & $\begin{array}{l}\text { ACV da emissão de gases de } \\
\text { efeito estufa na produção de } \\
\text { etanol }\end{array}$ & $\begin{array}{c}\text { European Environment } \\
\text { Agency (1997); United } \\
\text { States Environmental } \\
\text { Protection Agency (2006) }\end{array}$ & $\begin{array}{l}\text { Objetivo e escopo, análise de } \\
\text { inventário e interpretação de } \\
\text { resultados }\end{array}$ & ---- \\
\hline 17 & $\begin{array}{l}\text { Hansen, Seo e } \\
\text { Kulay }(2010)\end{array}$ & $\begin{array}{l}\text { ACV da produção de materiais } \\
\text { cerâmicos }\end{array}$ & ISO 14040 & $\begin{array}{l}\text { Objetivo e escopo, análise de } \\
\text { inventário, avaliação de impactos e } \\
\text { interpretação de resultados }\end{array}$ & SimaPro \\
\hline
\end{tabular}

A base de dados definida para esta pesquisa considerou primeiramente o evento e os periódicos da área de Engenharia de Produção tidos como oficiais pela ABEPRO ou, ainda, como no caso da Revista Gestão Industrial, reconhecidos com o selo da referida associação. Desta forma, à exceção dos periódicos da base SciELO Brasil, outros periódicos da área de Engenharia de Produção não foram considerados neste trabalho por, além de não serem reconhecidos pela ABEPRO, não conseguirem manter a regularidade de sua publicação. De fato pode ser que haja algum artigo nacional sobre ACV publicado em um periódico 
que não conste nas bases consultadas. Entretanto, ressaltamos que a base de dados em questão abrange os principais e mais qualificados meios nacionais de divulgação de trabalhos científicos. Ressalta-se que a extensão da consulta em toda a base SciELO Brasil permitiu encontrar artigos de áreas diversas, além da Engenharia de Produção.

Em relação aos congressos, o ENEGEP é de fato o principal evento da área de Engenharia de Produção, abrangendo trabalhos das suas diversas área de atuação, inclusive daquelas nas quais a temática ACV pode ser considerada. Isto foi comprovado pela predominância de trabalhos neste evento - 79\% dos artigos. De fato outros eventos da área de Engenharia de Produção poderiam ser considerados, como o Simpósio de Engenharia de Produção, SIMPEP. Entretanto, mesmo sendo o segundo principal evento da área, o SIMPEP não foi considerado por não ser um evento oficial realizado pela ABEPRO. Bases de dados de outras áreas do conhecimento poderiam ser consideradas, porém ficou definido que a pesquisa deveria ser realizada em bases on-line nacionais de acesso gratuito, para que os artigos pudessem ser lidos e avaliados minuciosamente, conforme apresentado.

É fato que existem trabalhos nacionais publicados em bases internacionais como conferências e periódicos. Entretanto, esses foram desconsiderados por estarem fora do escopo deste trabalho, que eram as bases nacionais. Desta forma, além de outros eventos, a Conferência Internacional sobre a Análise do Ciclo de Vida na América Latina, CILCA, não foi incluída por ter apenas uma edição no Brasil. Em relação ao já citado Congresso Brasileiro de Gestão do Ciclo de Vida, CBGCV, destaca-se que esse evento só possui um dos seus anais disponíveis para consulta de forma gratuita, dificultando assim uma plena avaliação dos trabalhos nele publicados.

A ACV é uma metodologia com grande potencial de aplicação na avaliação de impactos ambientais. A pesquisa realizada verificou um número considerável de trabalhos que abordam o tema, contudo, houve um menor número deles $(21 \%)$ que aplicaram efetivamente a metodologia da ACV. Ressalta-se que dos 17 trabalhos citados neste estudo, 11 foram publicados nos últimos quatro anos (2007, 2008, 2009 e 2010), refletindo a recente tendência de trabalhos sobre ACV, o que, novamente, demonstra a busca preferencial do ENEGEP para publicação, pois este possui 6 destes 11 trabalhos citados. Outro panorama que se pode construir é o das áreas de aplicação da metodologia, identificadas a partir desses trabalhos, que são: a agricultura, a agroindústria, a logística, usinas de cana-de-açúcar e etanol, as indústrias de eletrodomésticos, materiais odontológicos, farmacêutica, têxtil, de siderurgia, de refinaria de petróleo, de plástico, de cimento, de cerâmica e química.
A metodologia da ACV possui benefícios e limitações, relacionadas até mesmo pela ISO 14040 (2006). A compreensão das limitações e dos benefícios é importante para estabelecer a abrangência do estudo. Um exemplo, identificado no presente trabalho, seria que uma das características de pesquisas do tipo ACV é que essas são direcionadas a um produto específico de uma unidade produtiva, o qual é estudado com todas as suas peculiaridades. Outra questão é a etapa de avaliação de impactos ambientais, a qual é muito complexa e abrangente e que, numa situação real, envolve significativos estudos de equipes interdisciplinares e que representam todos os interesses sociais envolvidos na questão.

No entanto, entre seus benefícios, além do grande potencial de aplicação na avaliação de impactos ambientais, já citado, destaca-se que a ACV proporciona uma visão geral do real impacto causado pela fabricação de determinado produto e também determina as etapas críticas da produção que proporcionam altas descargas ambientais ou que consumem grandes quantidades de recursos naturais. Com isso pode-se comparar dois produtos ou processos e avaliar a melhor opção. Entretanto, nem sempre a melhor opção é a que possui melhor apelo ambiental, como o caso da ACV comparativa da produção de alface, na qual a produção orgânica é tão impactante quanto a intensiva e a hidropônica. Outro caso é o estudo comparativo das chapas de aço para eletrodomésticos, que mostrou que a chapa de aço carbono com aplicação do autolimpante não é a melhor opção do ponto de vista ambiental sendo, no entanto, uma configuração muito solicitada nos eletrodomésticos pelo mercado.

Desse modo, sugere-se uma maior difusão dessa metodologia através da abordagem direta do tema pela ABEPRO em seus fóruns de discussão como o ENEGEP, por meio da promoção de palestras, e/ou mini-cursos, ou incentivar a temática da ACV em seus periódicos, de modo à melhor divulgar e propagar a ACV para profissionais e pesquisadores das diversas regiões do pais, criando assim opções de bases científicas que poderão servir de referência para os trabalhos produzidos no Brasil.

\section{Referências}

ABARCA, C. D. G. et al. ISO 14000 - Análisis del Ciclo de Vida. In: ENCONTRO NACIONAL DE ENGENHARIA DE PRODUÇÃO, 18., 1997, Gramado. Anais... Gramado: ABEPRO, 1997.

ALMEIDA, A. R. C.; BRUNSTEIN, 1. Análise da rotulagem ambiental integrada ao ciclo de vida de produtos florestais. In: ENCONTRO NACIONAL DE ENGENHARIA DE PRODUÇÃO, 20, 1998, Niterói. Anais... Niterói: ABEPRO, 1998. 
ALMEIDA, J. et al. Incorporação da variável ambiental às decisões econômicas: um estudo de caso em uma processadora de aves. In: ENCONTRO NACIONAL DE ENGENHARIA DE PRODUÇÃO, 28., 2008, Rio de Janeiro. Anais... Rio de Janeiro: ABEPRO, 2008.

AZEVEDO, B. D.; CARMO, L. F. R. R. S.; FERREIRA, M. L. Análise e proposta de diretrizes para um sistema logístico de recolha e beneficiamento dos resíduos sólidos para áreas de favela em sintonia com os preceitos do GRSCM. In: ENCONTRO NACIONAL DE ENGENHARIA DE PRODUÇÃO, 30., 2010, São Carlos. Anais... São Carlos: ABEPRO, 2010.

AZEVEDO, P. S.; NOLASCO, A. M. Fatores de incorporação de requisitos ambientais no processo de desenvolvimento de produtos em indústrias de móveis sob encomenda. Ciência Rural, v. 39, n. 8, nov. 2009.

BARBOSA JÚNIOR, A. F. et al. Conceitos e Aplicações de ACV no Brasil. In: ENCONTRO NACIONAL DE ENGENHARIA DE PRODUÇÃO, 27., 2007, Foz do lguaçu. Anais... Foz do lguaçu: ABEPRO, 2007.

BARRETO, A. P. L. et al. Ciclo de vida dos produtos: certificação e rotulagem ambiental. In: ENCONTRO NACIONAL DE ENGENHARIA DE PRODUÇÃO, 27., 2007, Foz do lguaçu. Anais... Foz do lguaçu: ABEPRO, 2007.

BASTOS, A. L. A.; POSSAMAI, O. Modelo de apoio à decisão para fabricação baseado na avaliação da performance ambiental de produtos e dos objetivos estratégicos da organização. In: ENCONTRO NACIONAL DE ENGENHARIA DE PRODUÇÃO, 22., 2002, Curitiba. Anais... Curitiba: ABEPRO, 2002.

BAUER, P. E.; MACIEL FILHO, R. Incorporation of Environmental Impact Criteria in The Design and Operation of Chemical Processes. Brazilian Journal of Chemical Engineering, v. 21, n. 3, p. 405-414, 2004. http://dx.doi.org/10.1590/S0104-66322004000300005

BIAZIN, C. C.; GODOY, A. M. G. 0 selo verde: uma nova exigência internacional para as organizações. In: ENCONTRO NACIONAL DE ENGENHARIA DE PRODUÇÃO, 20., 2000, São Paulo. Anais... São Paulo: ABEPRO, 2000.

BORCHARDT, M. et al. Implementação do ecodesign: um estudo de caso na indústria eletrônica. In: ENCONTRO NACIONAL DE ENGENHARIA DE PRODUÇÃO, 27., 2007, Foz do lguaçu. Anais... Foz do lguaçu: ABEPRO, 2007.

BORCHARDT, M. et al. Considerações sobre ecodesign: um estudo de caso na indústria eletrônica automotiva. Ambiente \& Sociedade, v. 11, n. 2, p. 341-353, 2008.

BORCHARDT, M. et al. Ecodesign practices: understanding and prioritizing activities in the automotive industry. In: ENCONTRO NACIONAL DE ENGENHARIA DE PRODUÇÃo, 30., 2010, São Carlos. Anais... São Carlos: ABEPRO, 2010a.

BORCHARDT, M. et al. Reprojeto do contraforte: um caso de aplicação do ecodesign em manufatura calçadista. Produção, v. 20, n. 3, 2010 b.

CARDOSO, R. S. et al. Ciclo de vida do produto, tecnologia e sustentabilidade: breve análise da gestão ambiental de resíduos sólidos no Brasil. In: ENCONTRO NACIONAL DE ENGENHARIA DE PRODUÇÃO, 27, 2007, Foz do lguaçu. Anais... Foz do lguaçu: ABEPRO, 2007.

CHEHEBE, J. R. Análise do ciclo de vida de produtos: Ferramenta gerencial da ISO 14000. Rio de Janeiro: Qualitymark, 1997.
CORAL, E.; STROBEL, J. S.; SELIG, P. M. A competitividade empresarial no contexto dos indicadores de sustentabilidade corporativa. In: ENCONTRO NACIONAL DE ENGENHARIA DE PRODUÇÃO, 24., 2004, Florianópolis. Anais... Florianópolis: ABEPRO, 2004.

CULTRI, C. N.; SAAVEDRA, Y. M. B.; OMETTO, A. Indicadores sociais como subsídios para a avaliação social do ciclo de vida: uma revisão da literatura. In: ENCONTRO NACIONAL DE ENGENHARIA DE PRODUÇÃO, 30., 2010, São Carlos. Anais... São Carlos: ABEPRO, 2010.

CURRAN, M. A. Environmental life-cicle analysis. New York: McGraw-Hill, 1996.

DETONI, T. L.; PEREIRA, J. H. Relação entre as práticas de aprendizagem organizacional e a gestão ambiental: o caso Repar. In: ENCONTRO NACIONAL DE ENGENHARIA DE PRODUÇÃO, 25., 2005, Porto Alegre. Anais... Porto Alegre: ABEPRO, 2005.

EPA - ENVIRONMENTAL PROTECTION AGENCY; SETAC - SCIENCE APPLICATIONS INTERNATIONAL CORPORATION. Life Cycle Assessment: principles and practice. 2006. Disponivel em: <http://www.epa.gov/ ord/NRMRL/lcaccess/pdfs/600r06060.pdf>. Acesso em: abr. 2010.

EUROPEAN ENVIRONMENT AGENCY - EEA. Life cycle assessment: a guide to approaches, experiences and information sources. Copenhagen: EEA, 1997.

FARIAS FILHO, J. R. et al. A utilização de mídia interativa como ferramenta para o desenvolvimento do "pensamento sustentável” na construção Civil. In: ENCONTRO NACIONAL DE ENGENHARIA DE PRODUÇÃO, 25., 2005, Porto Alegre. Anais... Porto Alegre: ABEPRO, 2005.

FERREIRA, G. L. B.; FRANK, B. Avaliação do Ciclo de Vida: Uma aplicação prática para facilitar a escolha da melhor opção de projeto no desenvolvimento de novos produtos. In: ENCONTRO NACIONAL DE ENGENHARIA DE PRODUÇÃO, 20., 2000, São Paulo. Anais... São Paulo: ABEPRO, 2000.

FERROLl, P. C. M. et al. Projeto para o Meio Ambiente: Uma Nova Preocupação do Projetista. In: ENCONTRO NACIONAL DE ENGENHARIA DE PRODUÇÃO, 17., 1997, Gramado. Anais... Gramado: 1997.

GARCIA, J. C. C.; VON SPERLING, E. Emissão de gases de efeito estufa no ciclo de vida do etanol: estimativa nas fases de agricultura e industrialização em Minas Gerais. Engenharia Sanitária e Ambiental, v.15, n. 3, p. 217-222, 2010.

GIANNETTl, B. F. et al. Inventário de ciclo de vida da manufatura de seringas odontológicas. Produção, v. 18, n. 1, p. 155-169, 2008.

GIESTA, L. C.; SILVA, T. N. A educação ambiental e capital social em cooperativas de produção orgânica. In: ENCONTRO NACIONAL DE ENGENHARIA DE PRODUÇÃO, 26., 2006, Fortaleza. Anais... Fortaleza: 2006.

GONÇALVES, M. E.; MARINS, F. A. S. Logística Reversa numa Empresa de Laminação de Vidros: um estudo de caso. Gestão \& Produção, v. 13, n. 3, p. 397-410, 2006.

GRAEDEl, T. E.; ALlENBY, B. R.; COMRIE, P. R. Matrix Approaches to Abridged Life Cycle Assessment. Journal of Environmental Science \& Technology, v. 29, n. 3, p. 134A-139A, 1995

GRAF, R.; FlGUEIREDO, P. J. M. Uma aplicação da avaliação de ciclo de vida do produto no setor agrícola - comparação da produção de alface com as técnicas intensiva, 
hidropônica e orgânica. In: ENCONTRO NACIONAL DE ENGENHARIA DE PRODUÇÃO, 19., 1999, Rio de Janeiro. Anais... Rio de Janeiro: ABEPRO, 1999.

GUELERE FILHO, A. et al. Ecodesign: métodos e ferramentas. In: ENCONTRO NACIONAL DE ENGENHARIA DE PRODUÇÃO, 28., 2008, Rio de Janeiro. Anais... Rio de Janeiro: ABEPRO, 2008.

HANSEN, A. P.; SEO, E. S. M.; KULAY, L. A. Identificação de oportunidades de melhoria de desempenho ambiental em processo de produção de materiais cerâmicos via aplicação da técnica de avaliação de ciclo de vida (ACV). Revista Produção On Line, v. 10, n. 4, 2010.

IBRAHIM, G. D. et al. Análise de ciclo de vida de sacos plásticos produzidos por reciclagem: estudo de caso em Seropédica, RJ. In: ENCONTRO NACIONAL DE ENGENHARIA DE PRODUÇÃO, 27., 2007, Foz do lguaçu. Anais... Foz do lguaçu: ABEPRO, 2007.

INTERNATIONAL ORGANIZATION FOR STANDARDIZATION. ISO 14040: life cycle assessment. principles and framework. 1S0, 2006.

JABBOUR, C. J. C. Entre desafios e oportunidades: reflexões acerca da inserção da dimensão ambiental no currículo de engenharia de produção. In: ENCONTRO NACIONAL DE ENGENHARIA DE PRODUÇÃO, 26., 2006, Fortaleza. Anais... Fortaleza: ABEPRO, 2006.

JACOVELlı, S. J.; FIGUEIREDO, P. J. M. Avaliação de ciclo de vida simplificada aplicada a evolução de tornos. In: ENCONTRO NACIONAL DE ENGENHARIA DE PRODUÇÃO, 23., 2003, Ouro Preto. Anais... Ouro Preto: ABEPRO, 2003.

KRAEMER, M. E. P. Gestão ambiental na construção da imagem corporativa. In: ENCONTRO NACIONAL DE ENGENHARIA DE PRODUÇÃO, 24., 2004, Florianópolis. Anais... Florianópolis: ABEPRO, 2004.

LIBRELOTTO, L. 1.; FERROLl, P. C. M.; RADOS, G. V. Caracterização da sustentabilidade nas empresas de construção civil. In: ENCONTRO NACIONAL DE ENGENHARIA DE PRODUÇÃO, 23., 2003, Ouro Preto. Anais... Ouro Preto: ABEPRO, 2003.

LIMA, A. M. F. Avaliação do Ciclo de Vida no Brasil: inserção e perspectivas. 2007. Dissertação (Mestrado em Gerenciamento e Tecnologias Ambientais no Processo Produtivo)-Universidade Federal da Bahia, Salvador, 2007.

LOBO, Y. R. 0.; LIMA, P. C. Avaliação do Ciclo de Vida. In: Encontro NACIONAL DE ENGENHARIA DE PRODUÇÃO, 17., 1997, Gramado. Anais... Gramado: ABEPRO, 1997.

MACHADO, L. A.; HOFFMANN, R. Impactos ambientais da geração de energia elétrica no Rio Grande do Sul. In: ENCONTRO NACIONAL DE ENGENHARIA DE PRODUÇÃO, 23., 2003, Ouro Preto. Anais... Ouro Preto: ABEPRO, 2003.

MADRUGA, K.; NASCIMENTO, L. F.; ZAWISLAK, P. Produção Mais Limpa no Setor Automotivo e a Cadeia de Fornecedores do Rio Grande do Sul. In: ENCONTRO NACIONAL DE ENGENHARIA DE PRODUÇÃO, 19., 1999, Rio de Janeiro. Anais... Rio de Janeiro: ABEPRO, 1999.

MAGALHÃES, R. M. Crítica à Análise de Ciclo de Vida Orientada para o Meio Ambiente. In: ENCONTRO NACIONAL DE ENGENHARIA DE PRODUÇÃO, 18., 1998, Niterói. Anais... Niterói: ABEPRO, 1998.
MAKIYA, 1. K. Ecodesign e seu papel mobilizador de inclusão social e sustentabilidade. In: ENCONTRO NACIONAL DE ENGENHARIA DE PRODUÇÃO, 27., 2007, Foz do lguaçu. Anais... Foz do lguaçu: ABEPRO, 2007.

MONDARDO FILHO, M.; FRANK, B. Balanço ambiental de processos como ferramenta para a gestão ambiental. In: ENCONTRO NACIONAL DE ENGENHARIA DE PRODUÇÃo, 20., 2000, São Paulo. Anais... São Paulo: ABEPRO, 2000.

ÑAURI, M. H. C. et al. O. Sistemas de Informação e a Variável Ambiental: Gerenciando o Futuro das Empresas. In: ENCONTRO NACIONAL DE ENGENHARIA DE PRODUÇÃO, 17., 1997, Gramado. Anais... Gramado: ABEPRO, 1997.

NIGRI, E. M. et al. Comparando processos industriais e artesanais: uma aplicação da análise simplificada do ciclo de vida na produção de cachaça. In: ENCONTRO NACIONAL DE ENGENHARIA DE PRODUÇÃO, 30., 2010, São Carlos. Anais... São Carlos: ABEPRO, 2010.

NIGRI, E. M.; ROMEIRO FILHO, E.; ROCHA, S. D. F. Cimento tipo portland: uma aplicação da análise do ciclo de vida simplificada. In: ENCONTRO NACIONAL DE ENGENHARIA DE PRODUÇÃO, 19., 2009, Salvador. Anais... Salvador: ABEPRO, 2009.

OLIVEIRA, O. J.; SERRA, J. R. Benefícios e dificuldades da gestão ambiental com base na ISO $14001 \mathrm{em}$ empresas industriais de São Paulo. Produção, v. 20, n. 3, 2010.

PASCHOALINO, M. P.; MARCONE, G. P. S.; JARDIM, W. F. Os Nanomateriais e a Questão Ambiental. Química Nova, v. 33, n. 2, p. 421-430, 2010. http://dx.doi.org/10.1590/ S0100-40422010000200033

PASQUAL, D. L.; COSTA, C. A. G.; FERNANDES, F. C. Auditoria ambiental de conformidade legal: um enfoque à legislação ambiental federal e do Estado de Santa Catarina. In: ENCONTRO NACIONAL DE ENGENHARIA DE PRODUÇÃ̃, 26., 2006, Fortaleza. Anais... Fortaleza: ABEPRO, 2006.

PATZlAFF, J. 0.; KERN, A. P.; GONZÁlEZ, M. A. S. Projeto de edificações com apelo sustentável: elementos para a construção de um sistema de apoio à decisão. Revista Produção On Line, v. 10, n. 3, 2010.

PINTO, S. H. B. et al. Programas de Melhoria da Qualidade: Um Estudo em Construtoras de Grande Porte. In: ENCONTRO NACIONAL DE ENGENHARIA DE PRODUÇÃO, 27., 2007, Foz do lguaçu. Anais... Foz do lguaçu: ABEPRO, 2007.

POZZOBON, C. E.; RUPPENTHAL, J. E. Algumas implicações entre construção civil e meio ambiente. In: ENCONTRO NACIONAL DE ENGENHARIA DE PRODUÇÃO, 19., 1999, Rio de Janeiro. Anais... Rio de Janeiro: ABEPRO, 1999.

QUEIROZ, G. C.; GARCIA, E. E. C. Reciclagem de Sacolas Plásticas de Polietileno em Termos de Inventário de Ciclo de Vida. Polímeros, v. 20, n. 5, 2010.

RODRIGUES, C. R. B. et al. Sistemas Computacionais de apoio a Ferramenta Análise de Ciclo de Vida do Produto (ACV). In: ENCONTRO NACIONAL DE ENGENHARIA DE PRODUÇÃO, 28., 2008, Rio de Janeiro. Anais... Rio de Janeiro: ABEPRO, 2008.

ROQUE, V. F.; MOURA JÚNIOR, A. N. C. Análise de ciclo de vida como ferramenta para se alcançar Emissão Zero. In: ENCONTRO NACIONAL DE ENGENHARIA DE PRODUÇÃO, 17., 1997, Gramado. Anais... Gramado: ABEPRO, 1997 . 
RUY, M.; ALLIPRANDINI, D. H. Métodos para a avaliação ambiental de produtos no projeto conceitual: uma revisão da literatura. In: ENCONTRO NACIONAL DE ENGENHARIA DE PRODUÇÃO, 30., 2010, São Carlos. Anais... São Carlos: ABEPRO, 2010.

SANTOS, L. J. C.; TENÓRIO, J. A. S. Avaliação do ciclo de vida e custeio do ciclo de vida de evaporadores para usinas de açúcar. Revista Escola de Minas, v. 63, n. 1, p. 179-184, 2010. http://dx.doi.org/10.1590/ S0370-44672010000100030

SANTOS, L. M. M. Avaliação ambiental de processos industriais. 2. ed. São Paulo: Signus Editora, 2006.

SANTOS, S. Etapas de elaboração de projetos empresariais incluindo a variável ambiental. In: ENCONTRO NACIONAL DE ENGENHARIA DE PRODUÇÃO, 18., 1998, Niterói. Anais... Niterói: ABEPRO, 1998a.

SANTOS, S. S. R. Programas de Rotulagem Ambiental. In: ENCONTRO NACIONAL DE ENGENHARIA DE PRODUÇÃO, 18., 1998, Niterói. Anais... Niterói: ABEPRO, 1998b.

SElliTTO, M. A.; BORCHARDT, M.; PEREIRA, G. M. Modelagem para avaliação de desempenho ambiental em operações de manufatura. Gestão \& Produção, v. 17, n. 1, p. 95-109, 2010. http://dx.doi.org/10.1590/ S0104-530X2010000100008

SILVA, E. R. F.; SILVA, M. L. P. Aplicação dos Critérios de Produção Limpa nos Projetos Industriais. In: ENCONTRO NACIONAL DE ENGENHARIA DE PRODUÇÃO, 18., 1998, Niterói. Anais... Niterói: ABEPRO, 1998.

SILVA, 0. C. Eco-products and the environmental impact generated by logistical chains. In: Encontro NACIONAL DE ENGENHARIA DE PRODUÇÃO, 29., 2009, Salvador. Anais... Salvador: ABEPRO, 2009.

SILVA FILHO, J. C. G.; SICSÚ, A. B. Produção Mais Limpa: uma ferramenta da Gestão Ambiental aplicada às empresas nacionais. In: ENCONTRO NACIONAL DE ENGENHARIA
DE PRODUÇÃo, 23., 2003, Ouro Preto. Anais... Ouro Preto: ABEPRO, 2003.

TÁVORA, S. P.; QUELHAS, O. L. G. Óleos Lubrificantes Usados - Evolução das Responsabilidades pela Coleta/ Destinação e Alternativas para Aplicações: Uma Contribuição para a Tecnologia de Produção Mais Limpa. Revista Produção On Line, v. 3, n. 2, 2003.

TEIXEIRA, I. S.; TEIXEIRA, R. C. F. S. A Estratégia Empresarial e seus Reflexos sobre o Sistema de Gestão Ambiental. In: ENCONTRO NACIONAL DE ENGENHARIA DE PRODUÇÃO, 17., 1997, Gramado. Anais... Gramado: ABEPRO, 1997.

TOLEDO, T. P. A.; TURRIONI, J. B.; BALESTRASSI, P. P. Implantação do Sistema de Gestão Ambiental Segundo a ISO 14001: Um Estudo de Caso em uma Empresa do Sul de Minas Gerais. In: ENCONTRO NACIONAL DE ENGENHARIA DE PRODUÇÃO, 23., 2003, Ouro Preto. Anais... Ouro Preto: ABEPRO, 2003.

UGAYA, C. M. L.; HENSCHEL, J. Metodologia para identificação de aspectos ambientais significativos nos processos de refino de petróleo. In: ENCONTRO NACIONAL DE ENGENHARIA DE PRODUÇÃO, 24., 2004, Florianópolis. Anais... Florianópolis: ABEPRO, 2004.

ULIANA, R. B. Um panorama da evolução da abordagem e adoção da sustentabilidade na formação do engenheiro. In: ENCONTRO NACIONAL DE ENGENHARIA DE PRODUÇÃO, 28., 2008, Rio de Janeiro. Anais... Rio de Janeiro: ABEPRO, 2008.

UNITED STATES ENVIRONMENTAL PROTECTION AGENCY - USEPA. Life cycle assessment: principles and practice. Cincinnati: USEPA, 2006.

WILlERS, C. D. et al. Avaliação do Ciclo de Vida: uma investigação bibliográfica dos trabalhos sobre a metodologia. In: ENCONTRO NACIONAL DE ENGENHARIA DE PRODUÇÃO, 30., 2010, São Carlos. Anais... São Carlos: ABEPRO, 2010.

\title{
Life cycle assessment in Brazil: a research in the major national bases scientific
}

\begin{abstract}
The Life Cycle Assessment (LCA) is a methodology for evaluating the environmental impact of products and production systems, under the cradle to grave approach, namely, since the acquisition of raw materials to final disposal of materials. This work proposed to investigate the progress of the LCA studies in Brazil. A bibliographic research was performed on official or recognized scientific events and journals from the "Associação Brasileira de Engenharia de Produção" and SciELO Brazil Database. It was identified 80 articles, most of them from institutions of the South and Southeast Brazilian regions. The" Universidade de São Paulo" (USP) and the "Universidade Federal de Santa Catarina" (UFSC) had the largest number of publications among the 50 institutions identified. It was found that 17 articles effectively applied LCA methodology in a case study, and 11 used the methodology to evaluate the production process and 6 to compare materials or processes.
\end{abstract}

Keywords

Life cycle assessment. Impact assessment. 1 SO 14040. Bibliographic research. 